\title{
Oscillation of Nonlinear Differential Equations with Advanced Arguments
}

\author{
Hussain Ali Mohamad*
}

\author{
Sada F. Mohammed*
}

Awatif A. Hassan*

Date of acceptance 3/8/2008

\begin{abstract}
This paper is concerned with the oscillation of all solutions of the $n$-th order delay differential equation $x^{(n)}(t)+P(t) f(x(\tau(t)))=0, \quad n \geq 2$. The necessary and sufficient conditions for oscillatory solutions are obtained and other conditions for nonoscillatory solution to converge to zero are established.
\end{abstract}

\section{Introduction}

Consider the nonlinear differential equation of order $n$ with advanced argument of the type:-

$$
\begin{gathered}
x^{(n)}(t)+P(t) f(x(\tau(t)))=0, \\
n \geq 2 \quad, t \geq t_{0}
\end{gathered}
$$

Where the continuous function

$$
P:\left[t_{0}, \infty\right) \rightarrow R \text { is allowed to }
$$

oscillate, while the continuous functions $f: R \rightarrow R, \quad \tau:\left[t_{0}, \infty\right) \rightarrow R$ satisfies the

following conditions :

$\mathrm{H} 1: \tau(t)$ is continuous nondecreasing

$$
\tau(t)>t, \quad \lim _{t \rightarrow \infty} \tau(t)=\infty .
$$

$\mathrm{H} 2: f$ is nondecreasing such that

$$
u f(u)>0 \quad \text { for } \quad u \neq 0 .
$$

By a solution of eq. (1.1) we mean a function $x \in C^{1}\left(\left[t_{0}, \infty\right), R\right)$ which satisfies eq. (1.1) for all $t \geq t_{0}$ where $\sup \left\{|x(t)|: t \in\left[t_{x}, \infty\right)\right\}>0$. A solution is said to be non-oscillatory if it is eventually of constant sign otherwise is said oscillatory, eq. (1.1) is said oscillatory if all of its solutions are oscillatory. Many literatures studied the oscillation of eq.(1.1) of first and second order, and a few investigated the higher order. One may see the monographs due to Berezansky [1] Stavrovlakis [2] Ladde [3], Rath and Padhy [4], Kulenovic [5], Kiguradze,[6].

\section{Mean Results}

In this section we give some theorems describe the oscillatory behavior of the solutions of equation (1.1). 
Definition:- [see Kiguradze,[6] ]

Let $u(t) \in C^{n}([0, \infty), R)$ be of constant sign and let $u^{(n)}(t)$ be also of constant sign and not equivalent to zero in any interval

$[T, \infty), T \geq 0$ and $u(t) u^{(n)}(t) \leq 0 \quad$ then there exists $t_{0} \geq 0$ such that $u^{(i)}(t), \quad i=1,2, \ldots, n-1 \quad$ are of constant sign on $\left[t_{0}, \infty\right)$ and there exists an integer $k \in\{1,3,5, \ldots, n-1\}$ when $\mathrm{n}$ is even, or exists an integer $k \in\{0,2,4 \ldots, n-1\}$ when $\mathrm{n}$ is odd such that

$$
\begin{aligned}
& u(t) u^{(i)}(t)>0 \quad \text { for } \quad 0 \leq i \leq k \quad \text { on }\left[t_{0}, \infty\right) \\
& (-1)^{n+i-1} u(t) u^{(i)}(t)>0, \quad k+1 \leq i \leq n-1, t \geq t_{0}
\end{aligned}
$$

Such $u(t)$ is said to be of degree $k$

The following Lemma improve Lemma 6, Mohamad H. [7]

Lemma 1. Suppose that

$$
\delta\left\{u^{(n)}(t)+\delta P(t) f(u(\tau(t)))\right\} \operatorname{sgn} u(t) \leq 0,
$$

Where $\delta= \pm 1, P, f, \tau$ satisfies H1-H2

,$P(t) \geq 0$ and

$$
\int_{T}^{\infty} t^{2} P(t) d t=\infty
$$

Then the following statements are true:

1- Let $\delta=1$, if n even then every possible non-oscillatory solution of
(2.2) are of degree $n-1$. if $n$ odd then

every possible non- oscillatory solution of (2.2) are either of degree 0 or degree $n-1$.

2 - Let $\delta=-1$, if $n$ even then every possible non oscillatory solution of (2.2) are either of degree 0 or degree $n$. if $n$ odd then every possible non oscillatory solution of

(2.2) are of degree $n$.

Proof: See [7].

Other literatures study the case when

the coefficient is constant see [8]-[10], while Olach [11] study eq.(1.1) and gives some sufficient conditions for oscillation. Now we give the first result in this paper.

Theorem (1) : Suppose that $P(t) \geq 0$, and

$$
\int^{\infty} t^{n-1} P(t) d t=\infty
$$

Then every bounded solution of (1.1) is oscillatory if $n$ even, and every bounded solution are either oscillatory or $\lim _{t \rightarrow \infty} x(t)=0$ if $n$ is odd.

Proof: Let $x(t)$ is bounded nonoscillatory solution of eq. (1.1) on $\left[t_{0}, \infty\right)$.Without loss of generality let 
$x(t)>0$ on $\left[t_{0}, \infty\right)$, we have $x^{(n)}(t)=-P(t) f(x(\tau(t))) \leq 0$

Consider the equality

$$
\begin{aligned}
x^{(i)}(t)= & \sum_{i=1}^{n-1}(-1)^{i-j} \frac{(s-t)^{i-j}}{(i-j) !} x^{(i)}(s)+ \\
& \frac{(-1)^{n-j}}{(n-j-1) !} \int_{t}^{s}(u-t)^{n-j-1} x^{(n)}(u) d u
\end{aligned}
$$

Where $s \geq t \geq t_{0}$. By using eq. (1.1) the last equality leads to

$$
\begin{aligned}
& x^{(j)}(t)=\sum_{i=j}^{n-1}(-1)^{i-j} \frac{(s-t)^{i-j}}{(i-j) !} x^{(i)}(s)+ \\
& \frac{(-1)^{n-j+1}}{(n-j-1) !} \int_{t}^{s}(u-t)^{n-j-1} P(u) f(x(\tau(u))) d u
\end{aligned}
$$

let $n$ be even, since $x(t)$ is positive bounded and $x^{(n)}(t) \leq 0$ then $x(t)$ must be of degree 1 , let $j=1$ then the last equality reduce to :

$x^{\prime}(t) \geq \frac{1}{(n-2) !} \int_{t}^{\infty}(u-t)^{n-2} P(u) f(x(\tau(u))) d u \geq 0$

Integrate the last inequality from $t_{1}$ to

$t$ where $t \geq t_{1} \geq t_{0}$ we obtain

$x(t) \geq \frac{1}{(n-1) !} \int_{t_{1}}^{t}\left(u-t_{1}\right)^{n-1} P(u) f(x(\tau(u))) d u$

Since $x(t)$ is non-decreasing and bounded,

then

$\lim _{t \rightarrow \infty} x(t)=c>0, \quad x(t) \leq c \quad$ so we can

find $t_{2}$ large enough such that.

$$
\begin{array}{cl}
\frac{c}{2} \leq x(\tau(t)) \leq c & \text { and } \\
f(x(\tau(t))) \geq f\left(\frac{c}{2}\right)=c_{1}, & t \geq t_{2} \geq t_{1}
\end{array}
$$

, where $c_{1}$ is positive constant, as $t \rightarrow \infty$ we get from inequality (2.6)

$c \geq \frac{c_{1}}{(n-1) !} \int_{t_{2}}^{\infty}\left(u-t_{2}\right)^{n-1} P(u) d u$ which is a contradiction with (2.4).

Now let $n$ be odd, since $x^{(n)}(t) \leq 0$ then $x(t)$ must be of degree 0 which implies that $x(t)$ is non-increasing, from eq.(2.5) with $j=0$, we get :

$x\left(t_{1}\right) \geq \frac{1}{(n-1) !} \int_{t_{1}}^{t}\left(u-t_{1}\right)^{n-1} P(u) f(x(\tau(u))) d u$, $t \geq t_{1} \geq t_{0}$

Since $x^{\prime}(t) \leq 0$, and $x(t)$ is bounded, then $\lim _{t \rightarrow \infty} x(t)=c \geq 0, \quad x(t) \geq c$.

if $c \neq 0$ then $c>0$, we can find $t_{2} \geq t_{1}$ large enough such that $x(\tau(t)) \geq c$, and $f(x(\tau(t))) \geq f(c)=c_{1} \quad$ for $t \geq t_{2}$ then $x\left(t_{1}\right) \geq \frac{c_{1}}{(n-1) !} \int_{t_{2}}^{t}\left(u-t_{1}\right)^{n-1} P(u) d u$ as $t \rightarrow \infty$ we get a contradiction, so either $c=0$ or $x(t)$ is oscillatory.

Theorem (2): Suppose that $P(t) \leq 0$, and

$$
\int^{\infty} t^{n-1}|P(t)| d t=\infty,
$$

If $n$ even then every bounded solution of (1.1) are either oscillatory or 
$\lim _{t \rightarrow \infty} x(t)=0$, and if $n$ odd then every

bounded solution of (1.1) are oscillatory.

Proof: Let $x(t)$ is non oscillatory bounded solution of eq. (1.1), $t \geq t_{0}$

Without loss of generality let $x(t)>0$ on $\left[t_{0}, \infty\right)$, then

$$
x^{(n)}(t)=-P(t) f(x(\tau(t))) \geq 0
$$

Let $\mathrm{n}$ be even, since $x(t)$ is bounded and $x^{(n)}(t) \geq 0$, then $x(t)$ must be of degree 0 , which implies that $x(t)$ is non-increasing and bounded hence (2.5) reduce to

$$
\begin{aligned}
& x(t) \geq \frac{1}{(n-1) !} \int_{t}^{s}(u-t)^{n-1}|P(u)| f(x(\tau(u))) d u \geq 0 \\
& x\left(t_{1}\right) \geq \frac{1}{(n-1) !} \int_{t_{1}}^{s}\left(u-t_{1}\right)^{n-1}|P(u)| f(x(\tau(u))) d u
\end{aligned}
$$

$$
\geq 0, \quad t \geq t_{1}
$$

Let $\quad \lim _{t \rightarrow \infty} x(t)=c \geq 0, \quad x(t) \geq c, \quad$ if $c \neq 0$ then $c>0$, we can find $t_{2} \geq t_{1}$ large

enough such that $x(\tau(t)) \geq c$, and $f(x(\tau(t))) \geq f(c)=c_{1}>0 \quad$ for $t \geq t_{2} \quad$ then (2.7) implies to $x\left(t_{1}\right) \geq \frac{c_{1}}{(n-1) !} \int_{t_{2}}^{t}\left(u-t_{1}\right)^{n-1}|P(u)| d u$ as $t \rightarrow \infty$ we get a contradiction, so either $\mathrm{c}=0$ or $x(t)$ is oscillatory.

Let $n$ be odd, since $x^{(n)}(t) \geq 0$ then $x(t)$ must be of degree 1 which implies that $x(t)$ is non decreasing and bounded, from eq.(2.5) with $j=1$, we get :

$x^{\prime}(t) \geq \frac{1}{(n-2) !} \int_{t}^{\infty}(u-t)^{n-2}|P(u)| f(x(\tau(u))) d u$ Integrate the last inequality from $t_{1}$ to $t$ where $t \geq t_{1} \geq t_{0}$ we obtain $x\left(t_{1}\right) \geq \frac{1}{(n-1) !} \int_{t_{1}}^{s}\left(u-t_{1}\right)^{n-1}|P(u)| f(x(\tau(u))) d u$

$$
\geq 0, \quad t \geq t_{0}
$$

$\lim _{t \rightarrow \infty} x(t)=c>0, \quad x(t) \leq c$ so we can find $t_{2}$ large enough such that.

$$
\frac{c}{2} \leq x(\tau(t)) \leq c \quad \text { and }
$$

$f(x(\tau(t))) \geq f\left(\frac{c}{2}\right)=c_{1}, \quad t \geq t_{2} \geq t_{1}$ , where $c_{1}$ is positive constant, and as $t \rightarrow \infty$ we get from inequality (2.8) a contradiction.

\section{Theorem (3)}

Suppose that $P(t) \geq 0$, and (2.3) holds then if $n$ is even every solutions of (1.1) are oscillatory and if $n$ is odd every solutions of (1.1) are either oscillatory or $\lim _{t \rightarrow \infty} x(t)=0$.

Proof: Let $x(t)$ be non oscillatory solution of (1.1) and say $x(t)>0, t \geq t_{0}$ $x^{(n)}(t)=-P(t) f(x(\tau(t))) \leq 0, \quad$ then $x^{(i)}(t)$ are monotone $i=0,1, \ldots, n-1$ If $n$ even, then by Lemma 1 every non oscillatory solution of (1.1) are of degree $n-1$, integrate eq.(1.1) from $s$ to $t \quad s \leq t$ we get 


$$
\begin{gathered}
x^{(n-1)}(t)-x^{(n-1)}(s)=\int_{s}^{t} P(\xi) f(x(\tau(\xi))) d \xi, s<t \\
x^{(n-1)}(s) \geq \int_{s}^{t} P(\xi) f(x(\tau(\xi))) d \xi,
\end{gathered}
$$

consider the integral equality

$$
\begin{aligned}
\int_{t_{0}}^{t} \xi^{2} x^{(n)}(\xi) d \xi & =t^{2} x^{(n-1)}(t)-t_{0}^{2} x^{(n-1)}\left(t_{0}\right)-t x^{(n-2)}(t) \\
& +t_{0} x^{(n-2)}\left(t_{0}\right)+x^{(n-3)}(t)-x^{(n-3)}\left(t_{0}\right)
\end{aligned}
$$

$f\left(x\left(\tau\left(t_{0}\right)\right)\right) \int^{t} \xi^{2} P(\xi) d \xi \leq t_{0}^{2} x^{(n-1)}\left(t_{0}\right)-t^{2} x^{(n-1)}(t)+t x^{(n-2)}(t)$

$$
-t_{0} x^{(n-2)}\left(t_{0}\right)-x^{(n-3)}(t)+x^{(n-3)}\left(t_{0}\right)
$$

as $t \rightarrow \infty$ and apply (2.3) it follows that

$\lim _{t \rightarrow \infty}\left\{t x^{(n-2)}(t)-t^{2} x^{(n-1)}(t)-x^{(n-3)}(t)\right\}=\infty$

there is $t_{1} \geq t_{0} \quad$ such that $t x^{(n-2)}(t)-t^{2} x^{(n-1)}(t)-x^{(n-3)}(t) \geq 0, \quad t \geq t_{1}$

which implies that

$$
\begin{aligned}
& x^{(n-2)}(t) \geq t x^{(n-1)}(t), \quad t \geq t_{1} \\
& \int_{t_{1}}^{t}\left\{s x^{(n-2)}(s)-s^{2} x^{(n-1)}(s)-x^{(n-3)}(s)\right\} d s=t_{1}^{2} x^{(n-2)}\left(t_{1}\right)-t^{2} x^{(n-2)}(t) \\
&+3 t x^{(n-3)}(t)-3 t_{1} x^{(n-3)}\left(t_{1}\right)-4 x^{(n-4)}(t)+4 x^{(n-3)}\left(t_{1}\right)
\end{aligned}
$$

as $t \rightarrow \infty$ we get

$\lim _{t \rightarrow \infty}\left[3 t x^{(n-3)}(t)-t^{2} x^{(n-1)}(t)-4 x^{(n-4)}(t)\right]=\infty$

there is $t_{2} \geq t_{1}$ such that

$3 t x^{(n-2)}(t)-t^{2} x^{(n-1)}(t)-4 x^{(n-3)}(t) \geq 0, t \geq t_{2}$ which implies that

$$
3 x^{(n-2)}(t) \geq t x^{(n-1)}(t), \quad t \geq t_{2} \text {, follow }
$$

in this procures we get there is

$t_{n-2} \geq t_{n-3}$ such that

$$
\begin{aligned}
(2 n-3) t x(t)-t^{2} x^{\prime}(t)-(n-1)^{2} \int_{t_{n-2}}^{t} x(s) d s & \geq 0, \\
t & \geq t_{n-2}
\end{aligned}
$$

which implies that

$$
(2 n-3) x(t) \geq t x^{\prime}(t), \quad t \geq t_{n-2},
$$

$$
\text { then } x(t) \geq \frac{t^{n-1}}{\prod_{i=1}^{n-1}(2 i-1)} x^{(n-1)}(t),
$$

from the last inequality and (2.9) we obtain

$$
\begin{aligned}
x(s) & \geq \frac{s^{n-1}}{\prod_{i=1}^{n-1}(2 i-1)} x^{(n-1)}(s) \\
\geq & \frac{s^{n-1}}{\prod_{i=1}^{n-1}(2 i-1)} \int_{s}^{t} P(\xi) f(x(\tau(\xi))) d \xi \\
\geq & \frac{s^{n-1} f(x(\tau(s)))}{\prod_{i=1}^{n-1}(2 i-1)} \int_{s}^{t} P(\xi) d \xi \\
& \geq \frac{f(x(\tau(s)))}{\prod_{i=1}^{n-1}(2 i-1)} \int_{s}^{t} \xi^{n-1} P(\xi) d \xi
\end{aligned}
$$

$x\left(t_{n-2}\right) \geq \frac{f\left(x\left(\tau\left(t_{n-2}\right)\right)\right)}{\prod_{i=1}^{n-1}(2 i-1)} \int_{t_{n-2}}^{t} \xi^{n-1} P(\xi) d \xi$,

as $t \rightarrow \infty$ and according to (2.3) we get a contradiction

Let $\mathrm{n}$ be odd, by Lemma 1 every nonoscillatory solution of (1.1) are either of degree 0 or of degree n-1.

Suppose $x(t)$ is of degree 0 , then $x(t)$ is positive decreasing and so it is bounded and by Theorem 1 either $x(t)$ oscillatory or $\lim _{t \rightarrow \infty} x(t)=0$.

Suppose $x(t)$ is of degree $\mathrm{n}-1$, in this case the proof is similar when $n$ even. 


\section{Theorem (4)}

Assume that $P(t) \leq 0, \quad$ and $\frac{f(u)}{u} \geq M>0$

$\int_{T}^{\infty} t^{2}|P(t)| d t=\infty$,

$\limsup _{t \rightarrow \infty} \frac{M}{(n-1) !} \int_{\tau^{-1}(t)}^{t}(t-s)^{n-1}|P(s)| d s>1$,

If $n$ is even then every solution of (1.1) are either oscillatory or $\lim _{t \rightarrow \infty} x(t)=0$,

If $n$ is odd then every solution of (1.1) are oscillatory.

Proof : Let $x(t)$ be non-oscillatory solution of (1.1) and assume that

$$
\begin{gathered}
x(t)>0, t \geq t_{0} \text { hence } \\
x^{(n)}(t)=-P(t) f(x(\tau(t))) \leq 0, \quad \text { then } \\
x^{(i)}(t) \text { are monotone } i=0,1, \ldots, n-1
\end{gathered}
$$

Let $n$ be even, eq.(1.1) can be written as

$$
x^{(n)}(t)-|P(t)| f(x(\tau(t)))=0,
$$

so by Lemma 1 the only possible nonoscillatory solution of (1.1) are either of degree 0 or of degree n. Let $x(t)$ be of degree 0 ,then $x(t)$ is positive nonincreasing so it is bounded then by Theorem $1 x(t)$ is either oscillatory or $\lim _{t \rightarrow \infty} x(t)=0$.

Let $x(t)$ be of degree $\mathrm{n}$, using the equality where $\xi<t$ $x(t)=\sum_{i=0}^{n-1} \frac{(t-\xi)^{i}}{i !} x^{(i)}(\xi)+\frac{1}{(n-1) !} \int_{\xi}^{t}(t-s)^{n-1} x^{(n)}(s) d s$,

$x(t) \geq \frac{1}{(n-1) !} \int_{\xi}^{t}(t-s)^{n-1}|P(s)| f(x(\tau(s))) d s$,

Let $\tau(\xi) \geq t, \quad t \geq \xi \geq t_{1}$, hence

$x(\tau(\xi)) \geq \frac{1}{(n-1) !} \int_{\xi}^{t}(t-s)^{n-1}|P(s)| f(x(\tau(s))) d s$

$1 \geq \frac{1}{(n-1) ! x(\tau(\xi))} \int_{\xi}^{t}(t-s)^{n-1}|P(s)| f(x(\tau(s))) d s$

$\geq \frac{1}{(n-1) !} \int_{\tau^{-1}(t)}^{t}(t-s)^{n-1}|P(s)| \frac{f(x(\tau(s)))}{x(\tau(s))} d s$

$\geq \frac{M}{(n-1) !} \int_{\xi}^{t}(t-s)^{n-1}|P(s)| d s$,

This contradicts (2.10).

Let $\mathrm{n}$ be odd, by Lemma $1 x(t)$ must be of degree $n$, the prove is similar to the case when $n$ is even.

\section{Remarks and Examples :}

In this section we give some remarks and examples to illustrate the obtained results given in section 2

Remark1. If we use the condition $\frac{f(u)}{u} \geq M>0$ then the nondecreasing property of $f(u)$ needed not be necessary as we can see in Theorem 4.

Remark2. We can use the condition with $\quad \frac{f(u)}{u} \geq M>0 \quad$ to excluded the non-decreasing property of $f(u)$ in Theorem 1-Theorem 3.

Remark3. The conclusion of Theorem 4 remains true if we replace $(2,10)$ by the condition 
$\liminf _{t \rightarrow \infty} \int_{t}^{\tau(t)} \frac{(\tau(s)-h(s))^{n-1}}{(n-1) !}|q(s)| d s>\frac{1}{e}$

where $\mathrm{h}(\mathrm{t})$ is continuous function

such that $\tau(t) \geq h(t) \geq t$.

Example1. Consider the delay

differential equation:

$$
x^{\prime \prime \prime}(t)+\frac{6}{\left(2 t^{2}-1\right)^{2}} f(x(\tau(t)))=0, \quad t \geq 1
$$

with

$p(t)=\frac{6}{\left(2 t^{2}-1\right)^{2}}, \quad f(x(\tau(t)))=1-\frac{8 t^{2}}{2 t^{2}-1}$, satisfies $\mathrm{H} 1, \mathrm{H} 2$ and all the

conditions of Theorem 1, so all the

solutions of

equation (E.1) are either oscillatory or tends to zero as $t \rightarrow \infty$ for instance $x(t)=\ln \left(\frac{2 t-1}{2 t+1}\right)$ is such solution.

Example 2. Consider the delay differential equation:

$$
x^{\prime \prime}(t)+(a-\sin t) f(x(\tau(t)))=0, t \geq t_{0}
$$

\section{E.2}

with

$a \geq 1, \quad P(t)=1-\sin t, \quad f(x(\tau(t)))=-e^{-t}$ it is easy to see that all conditions of

Theorem 1 or Theorem 3 are hold so all solutions of equation (E.2) are either oscillatory or $\lim _{t \rightarrow \infty} x(t)=0$, for instance $x(t)=-e^{-t}\left(a+\frac{\sin t-\cos t}{4}\right)$ is such oscillatory solution of (E.2).

\section{Reference}

1. Berezansky, L. ,Bravermans1998. Some Oscillation Problems for second order linear Delay Differential Equations .J.Math. Analy.Appl. 220(1998): 719-740.

2. Kon, M., Sficas, Y. G., Stavrovlakis I. P.2000. Oscillation Criteria for delay equation. Proceeding of the American math. Society.128(16):2989-2997.

3. Ladde, G. S., Lakshmikantham, V. and Zhang, B. G..1987 Oscillation Theory Of Differential Equations With Deviating Arguments.Marcel Dekker Inc.New York And Basel.

4. Rath, R. N., Padhy I. N. 2004. Oscillation of Solutions of NonLinear Neutral Delay Differential Equations of Higher Order. Archivum math. 40(2004):359-366.

5. Kulenovic M. R. \& Grammatikopoulos M. K..1984. First order Functional Differential Inequalities With Oscillating Coefficients, Nonlinear Analysis, Theory Methods \&Applications,8(9):1043-1054.

6. Kiguradze, I. T. 1964 On the oscillation of solutions of the equation $\quad d^{m} u / d t^{m}+a(t)|u|^{n}$ sign $u=0$, mat. Sb. 65(1964):172-187.

7. Mohamad, Hussain 2000. Asymptotic behavior of $n$-th Order Linear Differential Equations Of Neutral Type. Proceedings of international Scientific conference .Bratislava, July 20-22 pp.105113.

8. Jianchu jiang: 2001. Oscillating criteria for first order non linear delay differential equations. Hiroshima Math. J. 31(2001):467476.

9. Ladas, G., Stavrovlakis I. P.1982.On Delay Differential Equations of Higher Order. Cana. Math. Bull.25(3):348-354. 
10. Seshadev Padhi 2005. Asymptotic behavior of solution to $\mathrm{N}$-th Order functional Differential Equations. Electronic journal of Diff. Eq.2005(65): pp.1-14.
11. Olach, R. 1983. Note on the Oscillation Of Differential Equation with advance arguments . Math. Slovoca.33(3):241-248.

\section{تذبذب المعادلات التفاضلية غير الخطية ذات المعاملات التقامية}

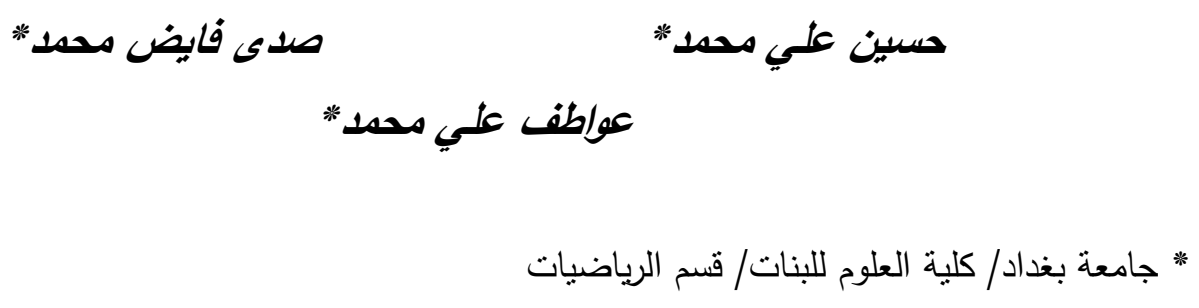

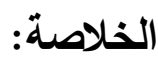

في هذا البحث قمنا باستخراج شروط كافية وضرورية لحلول المعادلات التفاضلية التباطئية ذات الرتب - $\quad x^{(n)}(t)+P(t) f(x(\tau(t)))=0, \quad n \geq 2$ من النو

كذلك اعطيت بعض الثروط الكافية للحلول غير المتذبذبة كي تكون منقاربة الى الصفر ـ كما واعطيت في البحث بعض الامتلة لتوضيح النتائج المستخرجة. 